\title{
Two efficient methods for solving fractional Lane-Emden equations with conformable fractional derivative
}

\author{
Adyan M. Malik ${ }^{1 *}$ and Osama H. Mohammed ${ }^{1}$
}

*Correspondence: adyan.muhammed@yahoo.com Dept. of Math. and Comp. Appl., Al-Nahrain University, Baghdad, 64055, Iraq

\begin{abstract}
In this paper, we introduce two reliable efficient approximate methods for solving a class of fractional Lane-Emden equations with conformable fractional derivative (CL-M) which are the so-called conformable Homotopy-Adomian decomposition method $(\mathrm{CH}-\mathrm{A})$ and conformable residual power series method (CRP). Furthermore, the proposed methods express the solutions of the non-linear cases of the CL-M in terms of fractional convergent series in which its components can be computed in an easy manner. Finally, the results are given by graphs for each case of the $C L-M$ at different values of $\alpha$ in order to demonstrate its accuracy, applicability, and efficiency.
\end{abstract}

Keywords: Fractional Lane-Emden equations, HAM, Residual power series

Subject Classification: Computational mathematics; Approximate methods; Numerical analysis

\section{Introduction}

The subject of fractional calculus can be considered as a generalization of the classical integer order calculus (derivative and integration), and due its important role in science and engineering, it has been gaining considerable attention of many authors and researchers [1-5]. The reader who is interested with the field of fractional calculus knows that there are many definitions of a fractional order derivative and they have been used to describe several real-life problems in many fields of sciences; the most important and famous ones are Riemann-Liouville, Grunwald-Letnikov, and Caputo derivatives [1, 2, 6-10]. Riemann-Liouville and Caputo use the integral in its construction, particularly the Cauchy integral formula with modifications. Hence, occasionally, we need complex computations to get the fractional derivative in the sense of Riemann-Liouville and Caputo. Furthermore, they do not satisfy the non-linear derivative rules as product, quotient, and chain rules. More recently, a new definition of fractional derivatives which is the so-called conformable fractional derivative has been introduced and attracted the attention of many researchers because it contains many characteristics that correspond to the usual derivative, particularly the Leibniz rules [11-16]. This definition is very simple and more welcome than other fractional definitions since it has been receiving a lot

(C) The Author(s). 2020 Open Access This article is licensed under a Creative Commons Attribution 4.0 International License which permits use, sharing, adaptation, distribution and reproduction in any medium or format, as long as you give appropriate credit to the original author(s) and the source, provide a link to the Creative Commons licence, and indicate if changes were made. The images or other third party material in this article are included in the article's Creative Commons licence, unless indicated otherwise in a credit line to the material. If material is not included in the article's Creative Commons licence and your intended use is not permitted by statutory regulation or exceeds the permitted use, you will need to obtain permission directly from the copyright holder. To view a copy of this licence, visit http://creativecommons.org/licenses/by/4.0/. 
of attention, many applications and phenomena can be modeled based on the CFDs, and it contains many interesting advantages such as the following: it is a local derivative that simulates the normal derivative because it depends on the limit in its formulation and it generalizes all concepts of ordinary calculus and can solve different fractional differential equations with all cases. In addition to this definition, there is another type of local derivatives called non-conformable fractional derivative, and for this purpose, the authors point out the publications [17-22]. In recent years, many authors have handled and studied the Lane-Emden equations because they were used to formulate lots of phenomena in physics and astrophysics [23-28].

The aim of this paper is to find the approximate solution of fractional LaneEmden equations with conformable fractional derivative (CL-M) using the conformable Homotopy-Adomian decomposition method ( $\mathrm{CH}-\mathrm{A})$ and conformable residual power series method (CRP); both of these methods are effective and easy to use for solving nonlinear CL-M, without linearization or discretization. The benefit of these techniques over the other methods is that they can be performed directly to the given problem by choosing an appropriate value for the initial guess approximations, and they also reduce the difficulty appearing in the computation of the complicated terms [29-36].

This paper is organized as follows: In the "Preliminaries" section, a preliminary introduction of the conformable fractional order derivative is presented. In "The $\mathrm{CH}-\mathrm{A}$ for solving $\mathrm{CL}-\mathrm{M}$ " section, we introduced the $\mathrm{CH}-\mathrm{A}$ for solving $\mathrm{CL}-$ $M$. The basic idea of the CRP technique for solving CL-M was given in the "A CRP for solving CL-M" section. Finally, a conclusion has been drawn.

\section{Preliminaries}

In this section, a brief introduction to the definition and properties of the conformable fractional derivative will be given [11, 12, 37-40].

Definition 1 Given a function $y:[0,+\infty) \rightarrow \mathbb{R}$, then the CFD of order $\alpha$ of $y$ is given by:

$$
C D^{\alpha}(y)(x)=\lim _{\epsilon \rightarrow 0} \frac{y\left(x+\in x^{1-\alpha}\right)-y(x)}{\epsilon}
$$

for all $x>0, \alpha \in(0,1)$. If $y$ is $\alpha$-differentiable in some $(0, a), a>0$ and $\lim _{x \rightarrow 0} y^{(\alpha)}(x)$ exist, then define $y^{(\alpha)}(0)=\lim _{x \rightarrow 0} y^{(\alpha)}(x)$.

Definition 2 The conformable integral of order $\alpha$ is defined by:

$$
C I_{t}^{\alpha}(y)(t)=\int_{a}^{t} y(x) x^{\alpha-1} d x, a \geq 0 .
$$

where the integral is the usual Riemann improper integral and $\alpha \in(0,1)$.

Lemma 1 Let $\alpha \in(0,1]$ and $f$, $g$ be $\alpha$-differentiable at a point $t>0$, then:

1. $C D^{\alpha} x^{p}=p x^{p-\alpha}$

2. If in addition $f$ is differentiable, then $C D^{\alpha} f(x)=x^{1-\alpha} \frac{d f}{d x}$ 
3. $C D^{\alpha}(\lambda)=0$, for all scalar function $y(x)=\lambda$

4. $C I^{\alpha}\left(x^{\mu}\right)=\frac{\Gamma(\alpha+\mu-n)}{\Gamma(\alpha+\mu+1)}, \mu \in \mathbb{R}, \alpha \in(n, n+1]$ for all $n \in N$

5. $C I^{\alpha}\left(C D^{\alpha} y(x)\right)=y(x)-\sum_{k=0}^{n} \frac{y^{k}(0)}{k !} x^{k}$

\section{The CH-A for solving CL-M}

In this section, we present the $\mathrm{CH}-\mathrm{A}$ for solving $\mathrm{CL}-\mathrm{M}$; the $\mathrm{CH}-\mathrm{A}$ is a hybrid method in which it combines between the HAM and the Adomian decomposition method (ADM). The calculations involved in this technique are more easy than the standard HAM especially when the non-linear term in the CL-M is decomposed by using the Adomian polynomials. To start the procedure, let us consider the general form of CL-M of order $\alpha>0$ :

$$
C D^{2 \alpha} y+\frac{2 \alpha}{x^{\alpha}}+C D^{\alpha} y+f(y)=0
$$

where $x>0$ and $0<\alpha \leq 1$.

To solve this problem using $\mathrm{CH}-\mathrm{A}$, first, rewrite Eq. (3), as follows:

$$
C D^{\alpha}\left[x^{2 \alpha} C D^{\alpha} y\right]=-x^{2 \alpha} f(y)
$$

Then, integrate Eq. (4) twice with respect to $x$, so the general fractional solution of Eq. (3) is given by:

$$
y(x)=c_{2}+\int c_{1} x^{-2 \alpha} d_{\alpha} x-\left[\int x^{-2 \alpha}\left[\int x^{2 \alpha} f(y) d_{\alpha} x\right] d_{\alpha} x\right]
$$

where $c_{1}$ and $c_{2}$ are constants and $d_{\alpha} x=x^{1-\alpha} d x$.

In order to solve Eq. (5) by means of HAM, we need to seek the auxiliary linear operator:

$$
\mathcal{L}[\Phi(x, q)]=\Phi(x, q)
$$

We now define the non-linear operator as:

$$
N[\Phi(x, q)]=\Phi(x, q)-c_{2}-\int c_{1} x^{-2 \alpha} d_{\alpha} x+\left[\int x^{-2 \alpha}\left[\int x^{2 \alpha} f(y) d_{\alpha} x\right] d_{\alpha} x\right]
$$

Consequently, the $m$ th-order $(m \geq 1)$ deformation equations can be expressed using the Adomian polynomials as:

$$
\left[y_{m}-\chi_{m} y_{m-1}\right]=\hbar H R_{m}\left(\vec{y}_{m-1}\right)
$$

where

$$
R_{m}\left(\vec{y}_{m-1}\right)=y_{m-1}-\left(1-\chi_{m}\right)\left(c_{2}+\int c_{1} x^{-2 \alpha} d_{\alpha} x-\left[\int x^{-2 \alpha}\left[\int x^{2 \alpha} A_{m} d_{\alpha} x\right] d_{\alpha} x\right]\right)
$$

Hence

$$
y_{m}=\chi_{m} y_{m-1}+\hbar H R_{m}\left(\vec{y}_{m-1}\right)
$$

Starting with an initial approximation:

$$
y_{0}=c_{2}+\int c_{1} x^{-2 \alpha} d_{\alpha} x
$$

So, we have:

$$
y_{m+1}(x)=-\left[\int x^{-2 \alpha}\left[\int x^{2 \alpha} A_{m} d_{\alpha} x\right] d_{\alpha} x\right], \quad m=0,1,2, \ldots .
$$


where

$$
\chi_{m}=\left\{\begin{array}{l}
0, \quad m \leq 1 \\
1, m>1
\end{array}\right.
$$

and $\left\{A_{m}\right\}_{m=0}^{+\infty}$ is the set of Adomian polynomials of $f(y)$ which is defined as follows:

$$
A_{m}=\frac{1}{m !} \frac{d^{m}}{d \theta^{m}}\left[f\left(\sum_{i=0}^{+\infty} \theta^{i} y_{i}\right)\right]_{\theta=0}, \quad m=0,1,2, \ldots .
$$

Finally, the exact solution of Eq. (3) can be calculated by:

$$
y(x)=\sum_{m=0}^{+\infty} y_{m}(x)
$$

and the $n$ th-order approximate solution of Eq. (3) is given by:

$$
y_{n}(x)=\sum_{m=0}^{n} y_{m}(x)
$$

Now, we examine some known and charming cases that have been resulting from Eq. (3) as follows:

Case $1 \operatorname{Set} f(y)=y^{k}, k=0,1,2, \ldots$ in Eq.(3), then the following equation for $x>0$ is called:

$$
C D^{2 \alpha} y+\frac{2 \alpha}{x^{\alpha}} C D^{\alpha} y+y^{k}=0, \quad 0<\alpha \leq 1, \quad k=0,1,2, \ldots .
$$

\section{The CL-M of the first kind.}

To solve this problem, we apply Eq. (13) to compute the set of Adomian polynomials $\left\{A_{k}\right\}_{k=0}^{+\infty}$ of the non-linear function $f(y)=y^{k}$.

Hence, according to Eq. (14), the general fractional solution of Eq. (16) is given as follows:

$$
y(x)=\sum_{m=0}^{+\infty} y_{m}(x)
$$

where $y_{0}$ is given by Eq. (11) and $y_{m}, m=1,2,3, \ldots$, is given by Eq. (12).

Problem 1 Set the initial conditions $y(0)=1$ and $y^{\prime}(0)=0$ into Eq. (16), then the general fractional solution of Eq. (16) in terms of Adomian polynomials is given by Eqs. (11), (12), and (14), respectively.

Problem 2 Set $k=0$ into Eq. (16), then we have:

$$
C D^{2 \alpha} y+\frac{2 \alpha}{x^{\alpha}} C D^{\alpha} y+1=0, \quad 0<\alpha \leq 1
$$

According to the initial conditions given in problem 1 and by integrating twice, then, the exact solution of this equation (see Fig. 1) can be obtained by :

$$
y(x)=1-\frac{x^{2 \alpha}}{6 \alpha^{2}}
$$


Problem 3 Set $k=1$ into Eq. (16) and by using the initial conditions $y(0)=1$ and $y^{\prime}(0)=0$, then we have:

$$
C D^{2 \alpha} y+\frac{2 \alpha}{x^{\alpha}} C D^{\alpha} y+y=0, \quad 0<\alpha \leq 1
$$

According to Eqs.(11) and (12), with $A_{0}=y_{0}, A_{1}=y_{1}, A_{2}=y_{2}$, etc., then the solution of Eq. (19) is (see Fig. 2):

$$
y(x)=1-\frac{x^{2 \alpha}}{6 \alpha^{2}}+\frac{x^{4 \alpha}}{120 \alpha^{4}}-\frac{x^{6 \alpha}}{5040 \alpha^{6}}
$$

Problem 4 Set $k=2$ into Eq. (16) and by using the initial conditions $y(0)=1$ and $y^{\prime}(0)=0$, then we have:

$$
C D^{2 \alpha} y+\frac{2 \alpha}{x^{\alpha}} C D^{\alpha} y+y^{2}=0, \quad 0<\alpha \leq 1
$$

Also by using Eqs.(11) and (12), with $A_{0}=y_{0}^{2}, A_{1}=2 y_{0} y_{1}, A_{2}=2 y_{0} y_{2}+y_{1}^{2}$, etc., then the solution of Eq. (21) is (see Fig. 3):

$$
y(x)=1-\frac{x^{2 \alpha}}{6 \alpha^{2}}+\frac{x^{4 \alpha}}{60 \alpha^{4}}-\frac{11 x^{6 \alpha}}{7560 \alpha^{6}}
$$

Case 2 Consider $f(y)=e^{y}$ as in Eq. (3), then the following equation for $x>0$ is called:

$$
C D^{2 \alpha} y+\frac{2 \alpha}{x^{\alpha}} C D^{\alpha} y+e^{y}=0, \quad 0<\alpha \leq 1
$$

The CL-M of the second kind.

To solve problem (23) using CH-A and according to Eqs.(11) and (12), with $A_{0}=$ 1, $A_{1}=y_{1}, A_{2}=y_{2}+\frac{1}{2} y_{1}^{2}, A_{3}=y_{3}+y_{1} y_{2}+\frac{1}{3 !} y_{1}^{3}, \ldots$, the solutions will be drawn as:

$$
y_{0}=0, y_{k+1}(x)=-\left[\int x^{-2 \alpha}\left[\int x^{2 \alpha} A_{k} d_{\alpha} x\right] d_{\alpha} x\right], \quad k=0,1,2, \ldots
$$

Therefore, the general fractional power series solution is (see Fig. 4) given by:

$$
y(x)=-\frac{x^{2 \alpha}}{6 \alpha^{2}}+\frac{x^{4 \alpha}}{120 \alpha^{4}}-\frac{x^{6 \alpha}}{1890 \alpha^{6}}
$$

\section{A CRP for solving CL-M}

The implementation of the CRP will be described in this segment in order to get the fractional power series solution of the CL-M represented by Eq. (3) subject to the initial conditions:

$$
y\left(x_{0}\right)=a, y^{\prime}\left(x_{0}\right)=b
$$

The solution of Eq. (3) with respect to Eq. (24) is proposed by CRP as a fractional power series expansion about the initial point $x=x_{0}$ as follows:

$$
y(x)=\sum_{m=0}^{+\infty} y_{m}(x)
$$

where $y_{m}$ is expressed by $y_{m}=c_{m} \frac{\left(x-x_{0}\right)^{m \alpha}}{\alpha^{m} m !}, m=0,1,2, \ldots$.

Clearly, for the case $m=0,1$, we have from the initial conditions given by Eq. (26) that $c_{0}=a$ and $c_{1}=b$. 
By truncating the summation into Eq. (27), we get the $k$ th approximate series:

$$
y^{k}(x)=\sum_{m=0}^{k} c_{m} \frac{\left(x-x_{0}\right)^{m \alpha}}{\alpha^{m} m !}
$$

For the convergence of the fractional power series Eq. (28), we advise to see [41].

For simplification and before employing the CRP, Eq. (3) can be written in the form:

$$
x^{\alpha} C D^{2 \alpha} y+2 \alpha C D^{\alpha} y+x^{\alpha} f(y)=0
$$

Now, to evaluate $c_{m}$ that appears in the series expansion given by Eq. (28), substituting $y^{k}(x)$ into Eq. (29), we obtain the following $k$ th residual function:

$$
\begin{aligned}
\operatorname{Res}^{k}(x)= & x^{\alpha} \sum_{m=2}^{k} \alpha^{2} m(m-1) c_{m} \frac{\left(x-x_{0}\right)^{(m-2) \alpha}}{\alpha^{m} m !}+2 \alpha \sum_{m=1}^{k} \alpha m c_{m} \frac{\left(x-x_{0}\right)^{(m-1) \alpha}}{\alpha^{m} m !} \\
& +x^{\alpha} f\left(\sum_{m=0}^{k} c_{m} \frac{\left(x-x_{0}\right)^{m \alpha}}{\alpha^{m} m !}\right)
\end{aligned}
$$

Then, $\operatorname{Res}^{\infty}(x)=\lim _{k \rightarrow \infty} \operatorname{Res}^{k}(x)$ clearly $\operatorname{Res}^{\infty}(x)=0$ for each $x \in\left[x_{0}, x_{0}+a\right]$

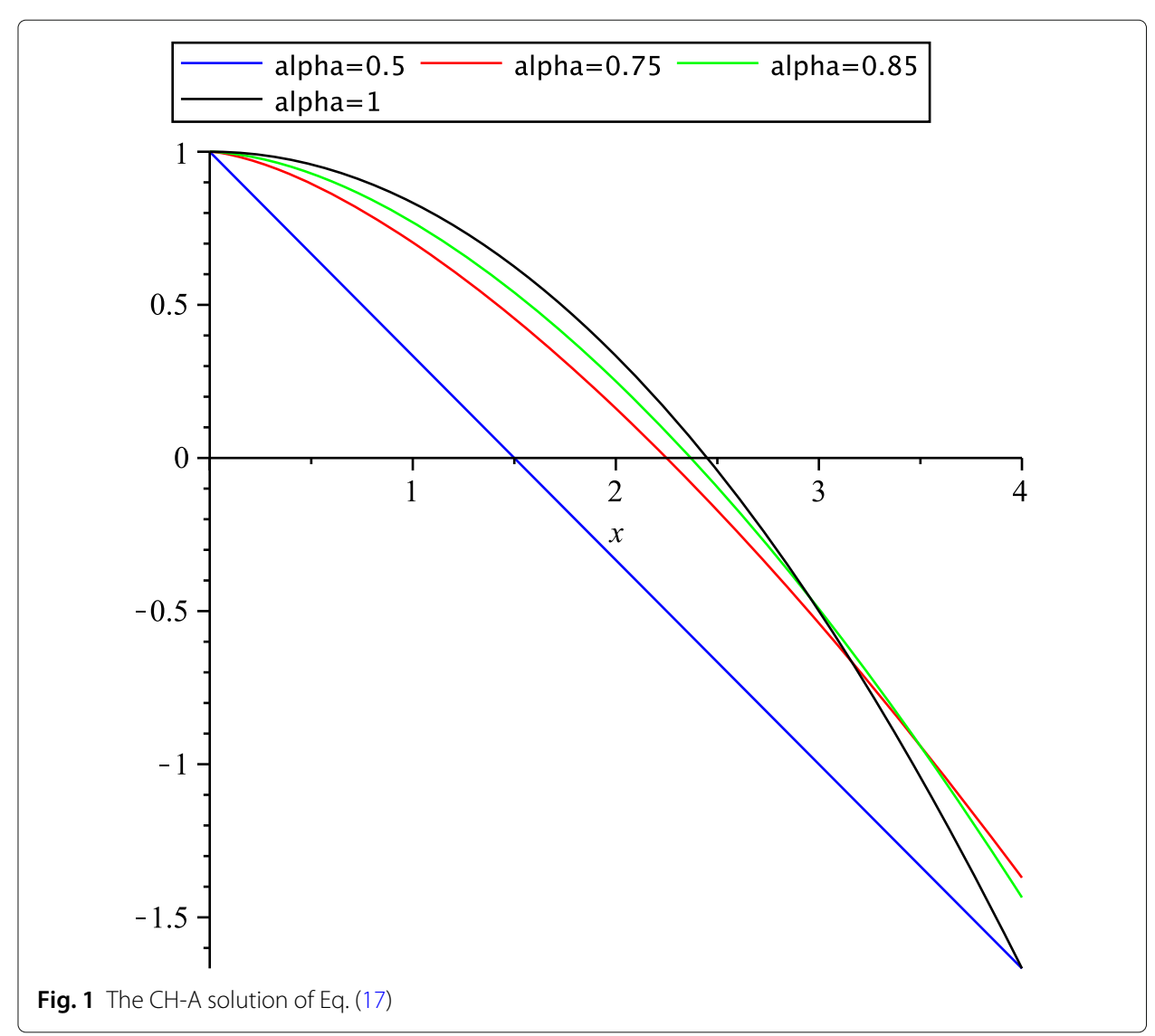


Furthermore, $C D^{(k-1) \alpha} \operatorname{Res}^{k}\left(x_{0}\right)=0, k=1,2,3, \ldots, k$ which is coming from lemma 1 (property 3 ) and this is a basic rule to compute the coefficients $c_{m}, m=2,3, \ldots, k$.

However, finding $c_{m}$ demands to solve the algebraic expression:

$$
C D^{(k-1) \alpha} \operatorname{Res}^{k}\left(x_{0}\right)=0, x \in\left[x_{0}, x_{0}+a\right]
$$

In this manner, all the desired coefficients $c_{m}$ will be found.

For solving Eq. (3) using CRP, different cases for Eq. (3) will be discussed as follows:

Case (i) Consider Eq. (17), subject to the initial conditions $y(0)=1$ and $y^{\prime}(0)=0$, according to the initial conditions given above, and by using Eq. (30), hence, we have $c_{0}=1, c_{1}=0, c_{2}=\frac{-1}{3}, c_{m}=0$ for all $m \geq 3$, substituting these values into Eq. (28) then the general fractional series solution in this case can be obtained by:

$$
y^{\infty}(x)=1-\frac{x^{2 \alpha}}{6 \alpha^{2}}
$$

The nature of CRP solution of Eq.(17) is given in Fig. 1.

Case (ii) In this case, the problem under consideration combines Eq. (21) together with the initial conditions $y(0)=1$ and $y^{\prime}(0)=0$ also according to the given initial conditions and Eq.(30); then, we have $c_{0}=1, c_{1}=0, c_{2}=\frac{-1}{3}, c_{3}=0, c_{4}=$ $\frac{2}{5}, c_{5}=0, \ldots$; then, the fractional power series solution is:

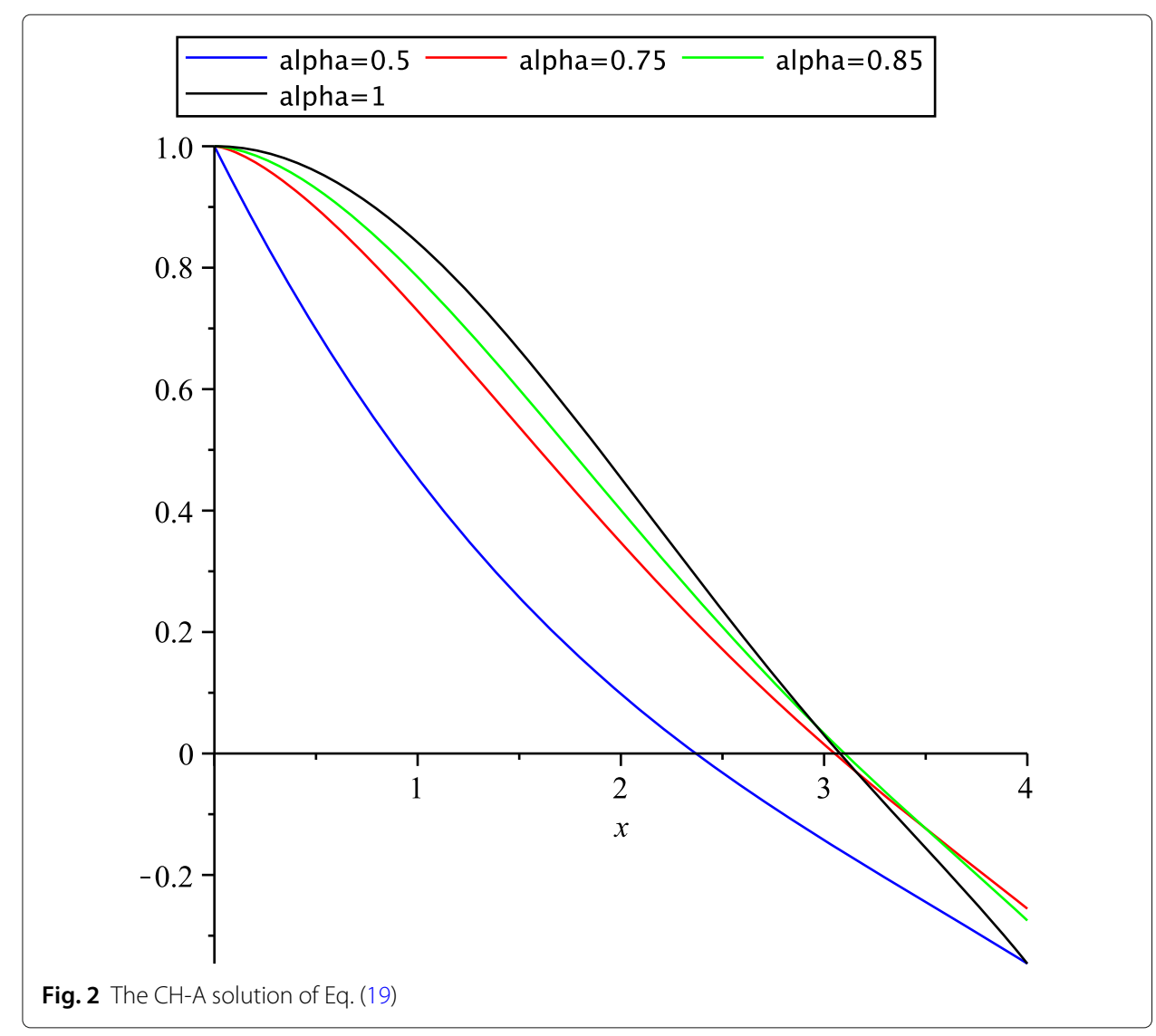




$$
y^{6}(x)=1-\frac{x^{2 \alpha}}{6 \alpha^{2}}+\frac{x^{4 \alpha}}{60 \alpha^{4}}-\frac{11 x^{6 \alpha}}{7560 \alpha^{6}}
$$

Also, the nature of the CRP solution of Eq. (21) is presented in Fig. 3.

Case (iii) Consider the problem given by Eq. (23) with respect to the initial conditions $y(0)=0$ and $y^{\prime}(0)=0$, then after taking the initial conditions in consideration and by employing Eq. (30), we get $c_{0}=c_{1}=0, c_{2}=\frac{-1}{3}, c_{3}=0, c_{4}=\frac{1}{5}$, $c_{5}=0, \ldots$; thus, the fractional power series solution in this case is:

$$
y^{6}(x)=-\frac{x^{2 \alpha}}{6 \alpha^{2}}+\frac{x^{4 \alpha}}{120 \alpha^{4}}-\frac{x^{6 \alpha}}{1890 \alpha^{6}}
$$

The quality of the CRP solution of Eq. (23) is given in Fig. 4.

\section{Conclusions}

In this paper, we applied $\mathrm{CH}$-A and $\mathrm{CRP}$ to find the approximate analytic solution of some classes of CL-M in terms of infinite fractional power series. The proposed methods introduced an easy way to compute the components of the solution that have been converging rapidly to the exact solution. The results obtained by $\mathrm{CH}-\mathrm{A}$ and CRP prove that these algorithms are highly effective and convenient in non-linear cases of the CL-M and can be employed to examine a wide class of non-linear fractional mathematical models.

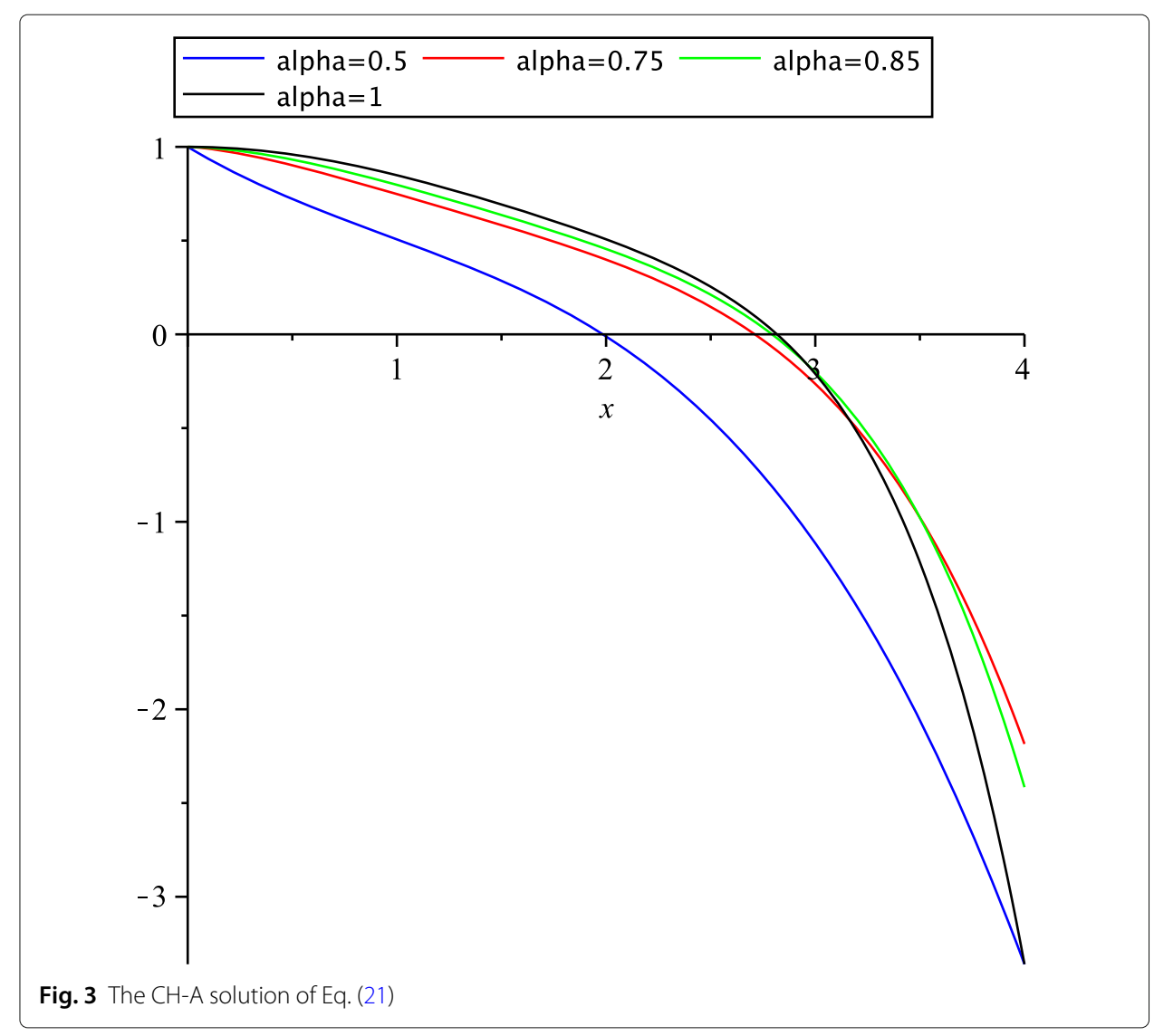




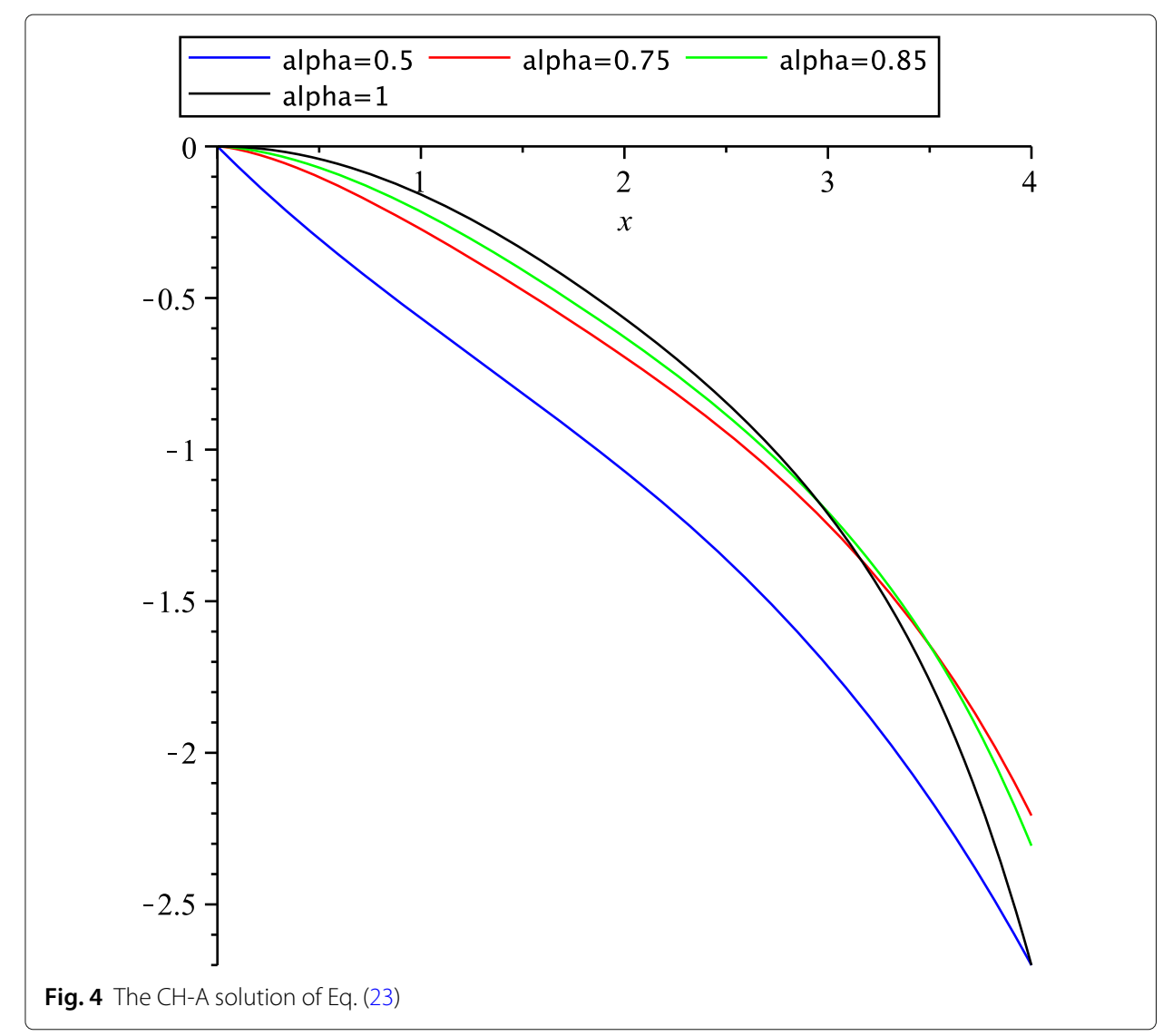

Acknowledgements

The authors are very grateful to the reviewers for their careful reading of the paper and for their comments and suggestions which have improve the paper.

\section{Authors' contributions}

The corresponding author analyzed and wrote the paper. The co-author raised the first idea and edited and analyzed the paper again in the well-organized form. Both authors read and approved the final manuscript.

\section{Funding}

Not applicable.

\section{Availability of data and materials}

All data generated or analyzed during this study are included in this paper.

\section{Competing interests}

The authors declare that they have no competing interests.

Received: 17 April 2020 Accepted: 23 July 2020

Published online: 18 August 2020

\section{References}

1. Podlubny, I.: Fractional Differential Equations : an Introduction to Fractional Derivatives, Fractional Differential Equations, to Methods of Their Solution and Some of Their Applications. Academic Press, San Diego (1999)

2. Kilbas, A. A.: Theory and Applications of Fractional Differential Equations. Elsevier, Amsterdam Boston (2006)

3. Mohammed, O. H.: A direct method for solving fractional order variational problems by hat basis functions. Ain Shams Eng. J. 9(4), 1513-1518 (2018). https://doi.org/10.1016/j.asej.2016.11.006

4. Mohammed, O. H., Malik, A. M.: A modified computational algorithm for solving systems of linear integro-differential equations of fractional order. J. King Saud Univ. Sci. 31(4), 946-955 (2019). https://doi.org/10.1016/j.jksus.2018.09.005

5. Abdo, M. S., Shah, K., Wahash, H. A., Panchal, S. K.: On a comprehensive model of the novel coronavirus (COVID-19) under mittag-leffler derivative. Chaos Solitons Fractals. 135, 109867 (2020). https://doi.org/10.1016/j.chaos.2020.109867

6. Ali, S., Bushnaq, S., Shah, K., Arif, M.: Numerical treatment of fractional order cauchy reaction diffusion equations. Chaos Solitons Fractals. 103, 578-587 (2017). https://doi.org/10.1016/j.chaos.2017.07.016 
7. Ali, S., Shah, K., Jarad, F.: On stable iterative solutions for a class of boundary value problem of nonlinear fractional order differential equations. Math. Methods Appl. Sci. 42(3), 969-981 (2018). https://doi.org/10.1002/mma.5407

8. Khan, H., Khan, A., Chen, W., Shah, K.: Stability analysis and a numerical scheme for fractional klein-gordon equations. Math. Methods Appl. Sci. 42(2), 723-732 (2018). https://doi.org/10.1002/mma.5375

9. Kamal, S.: Using a numerical method by omitting discretization of data to study numerical solutions for boundary value problems of fractional order differential equations. Math. Methods Appl. Sci. 42(18), 6944-6959 (2019). https:// doi.org/10.1002/mma.5800

10. Shah, K., Alqudah, M. A., Jarad, F., Abdeljawad, T.: Semi-analytical study of pine wilt disease model with convex rate under caputo-febrizio fractional order derivative. Chaos Solitons Fractals. 135, 109754 (2020). https://doi.org/10. 1016/j.chaos.2020.109754

11. Khalil, R., Al Horani, M., Yousef, A., Sababheh, M.: A new definition of fractional derivative. J. Comput. Appl. Math. 264 65-70 (2014). https://doi.org/10.1016/j.cam.2014.01.002

12. Abdeljawad, T.: On conformable fractional calculus. J. Comput. Appl. Math. 279, 57-66 (2015). https://doi.org/10. 1016/j.cam.2014.10.016

13. Dedovic, N., Birgani, O. T., Chandok, S., Radenovic, S.: A note on some recent results of the conformable fractional derivative. Adv. Theory Nonlinear Anal. Appl., 11-17 (2018). https://doi.org/10.31197/atnaa.482525

14. Todorčević, V.: Subharmonic behavior and quasiconformal mappings. Anal. Math. Phys. 9(3), 1211-1225 (2019). https://doi.org/10.1007/s13324-019-00308-8

15. Manojlovic, V.: On conformally invariant extremal problems. Appl. Anal. Discret. Math. 3(1), 97-119 (2009). https:// doi.org/10.2298/aadm0901097m

16. Shanmugam, T., Muthiah, M., Radenović, S.: Existence of positive solution for the eighth-order boundary value problem using classical version of leray-schauder alternative fixed point theorem. Axioms. 8(4), 129 (2019). https:// doi.org/10.3390/axioms8040129

17. Fleitas, A., Mendez-Bermudez, J. A., Valdés, J. E. N., Almira, J. M. S.: On fractional liénard-type systems. Rev. Mex. Fís. 65(6 Nov-Dec), 618 (2019). https://doi.org/10.31349/revmexfis.65.618

18. Fleitas, A., Gómez-Aguilar, J. F., Valdés, J. E. N, Rodríguez, J. M., M., S. J.: Analysis of the local drude model involving the generalized fractional derivative. Optik. 193, 163008 (2019). https://doi.org/10.1016/j.ijleo.2019.163008

19. Guzmán, P. M., Nápoles Valdés, J. E.: A note on the oscillatory character of some non conformable generalized lienard system. Advanced Mathematical Models and Applications (2019)

20. Guzmán, P. M., Lugo Motta-Bittencart, L. M., Nápoles Váldes, J. E.: A new definition of a fractional derivative of local type. J. Math. Anal. (2018)

21. Martinéz, F., Mohammed, P. O., Nápoles Váldes, J. E.: Non-conformable fractional laplace transform. Kragujevac J. Math. (2019)

22. Nápoles Váldes, J. E., Guzmán, P. M.: Some new results on nonconformable fractional calculus. Adv. Dyn. Syst. Appl. (2018)

23. Wang, X., Zhang, J.: Non-existence of positive solutions to nonlocal lane-emden equations. J. Math. Anal. Appl. 488(1), 124067 (2020). https://doi.org/10.1016/j.jmaa.2020.124067

24. Dizicheh, A. K., Salahshour, S., Ahmadian, A., Baleanu, D.: A novel algorithm based on the legendre wavelets spectral technique for solving the lane-emden equations. Appl. Numer. Math. 153, 443-456 (2020). https://doi.org/10.1016/ j.apnum.2020.02.016

25. Hadian-Rasanan, A. H., Rahmati, D., Gorgin, S., Parand, K.: A single layer fractional orthogonal neural network for solving various types of lane-emden equation. New Astron. 75, 101307 (2020). https://doi.org/10.1016/j.newast. 2019.101307

26. Singh, R., Garg, H., Guleria, V.: Haar wavelet collocation method for lane-emden equations with dirichlet, neumann and neumann-robin boundary conditions. J. Comput. Appl. Math. 346, 150-161 (2019). https://doi.org/10.1016/j. cam.2018.07.004

27. Al-Zhour, Z., Al-Mutairi, N., Alrawajeh, F., Alkhasawneh, R.: Series solutions for the laguerre and lane-emden fractional differential equations in the sense of conformable fractional derivative. Alex. Eng. J. 58(4), 1413-1420 (2019). https:// doi.org/10.1016/j.aej.2019.11.012

28. Soliman, M. A.: Approximate solution for the lane-emden equation of the second kind in a spherical annulus. J. King Saud Univ. Eng. Sci. 31(1), 1-5 (2019). https://doi.org/10.1016/j.jksues.2017.02.002

29. Hussain, S., Shah, A., Ayub, S., Ullah, A.: An approximate analytical solution of the allen-cahn equation using homotopy perturbation method and homotopy analysis method. Heliyon. 5(12), 03060 (2019). https://doi.org/10. 1016/j.heliyon.2019.e03060

30. Naik, P. A., Zu, J., Ghoreishi, M.: Estimating the approximate analytical solution of HIV viral dynamic model by using homotopy analysis method. Chaos Solitons Fractals. 131, 109500 (2020). https://doi.org/10.1016/j.chaos.2019.109500

31. Yépez-Martínez, H., Gómez-Aguilar, J. F.: A new modified definition of caputo-fabrizio fractional-order derivative and their applications to the multi step homotopy analysis method (MHAM). J. Comput. Appl. Math. 346, 247-260 (2019). https://doi.org/10.1016/j.cam.2018.07.023

32. Odibat, Z:: On the optimal selection of the linear operator and the initial approximation in the application of the homotopy analysis method to nonlinear fractional differential equations. Appl. Numer. Math. 137, 203-212 (2019). https://doi.org/10.1016/j.apnum.2018.11.003

33. Dubey, V. P., Kumar, R., Kumar, D.: A reliable treatment of residual power series method for time-fractional black-scholes European option pricing equations. Phys. A Stat. Mech. Appl. 533, 122040 (2019). https://doi.org/10. 1016/j.physa.2019.122040

34. Bayrak, M. A., Demir, A.: A new approach for space-time fractional partial differential equations by residual power series method. Appl. Math. Comput. 336, 215-230 (2018). https://doi.org/10.1016/j.amc.2018.04.032

35. Az-Zo'bi, E. A., Yıldırım, A., AlZoubi, W. A.: The residual power series method for the one-dimensional unsteady flow of a van der waals gas. Phys. A Stat. Mech. Appl. 517, 188-196 (2019). https://doi.org/10.1016/.jphysa.2018.11.030

36. Şenol, M., Alquran, M., Kasmaei, H. D.: On the comparison of perturbation-iteration algorithm and residual power series method to solve fractional zakharov-kuznetsov equation. Results Phys. 9, 321-327 (2018). https://doi.org/10. 1016/j.rinp.2018.02.056 
37. Abu Hammad, M., Khalil, R.: Conformable fractional heat differential equation. Int. J. Pure Appl. Math. 94(2) (2014). https://doi.org/10.12732/ijpam.v94i2.8

38. El-Ajou, A., Al-Zhour, Z., Oqielat, M., Momani, S., Hayat, T.: Series solutions of nonlinear conformable fractional KdVburgers equation with some applications. Eur. Phys. J. Plus. 134(8) (2019). https://doi.org/10.1140/epjp/i2019-12731-x

39. Oqielat, M. N., El-Ajou, A., Al-Zhour, Z., Alkhasawneh, R., Alrabaiah, H.: Series solutions for nonlinear time-fractional schr?dinger equations: Comparisons between conformable and caputo derivatives. Alex. Eng. J. (2020). https://doi. org/10.1016/j.aej.2020.01.023

40. Abdeljawad, T., Al-Mdallal, Q. M., Jarad, F.: Fractional logistic models in the frame of fractional operators generated by conformable derivatives. Chaos Solitons Fractals. 119, 94-101 (2019). https://doi.org/10.1016/j.chaos.2018.12.015

41. El-Ajou, A., Arqub, O., Zhour, Z., Momani, S.: New results on fractional power series: theories and applications. Entropy. 15(12), 5305-5323 (2013). https://doi.org/10.3390/e15125305

\section{Publisher's Note}

Springer Nature remains neutral with regard to jurisdictional claims in published maps and institutional affiliations.

\section{Submit your manuscript to a SpringerOpen ${ }^{\circ}$ journal and benefit from:}

- Convenient online submission

- Rigorous peer review

- Open access: articles freely available online

- High visibility within the field

- Retaining the copyright to your article

Submit your next manuscript at $\boldsymbol{\triangleright}$ springeropen.com 